\title{
HIF-2a - a mediator of stem cell altruism?
}

\author{
Eoin P Cummins*
}

\begin{abstract}
Human embryonic stem cells (hESCs) have been reported to confer cytoprotection in the context of tissue injury. This is somewhat counterintuitive given that microenvironmental factors such as hypoxia and oxidative stress may activate p53 and result in death and differentiation of these hESCs. In this article, we discuss a novel mechanism through which hESCs can be re-programmed (through exposure to hypoxia/ oxidative stress) to transiently suppress p53, enhance 'stemness', and exist in a highly cytoprotective and undifferentiated state.
\end{abstract}

Hypoxia is the situation that arises when the cellular demand for oxygen to produce sufficient amounts of ATP to maintain cellular function exceeds the supply. This situation can occur in a number of ways, including exposure to high altitude [1], increased metabolic activity, disruption of vascular supply [2], inflammation/injury [3], and tumorigenesis [4]. Over the course of evolution, organisms have developed the ability to adapt to conditions of hypoxia and elicit non-transcriptional and transcriptional responses in order to promote adaptation and survival. The alpha subunits of the hypoxia-inducible factor transcription factor family (HIF- $1 \alpha$ and HIF- $2 \alpha$ ) have been identified as the master regulators of the cellular response to hypoxia $[5,6]$. Together, these proteins regulate the expression of hundreds of genes involved in erythropoiesis, angiogenesis, vasodilation, glucose transport, glycolysis, iron metabolism, barrier function, and stem cell function in a multi-faceted adaptation to low oxygen.

HIF- $1 \alpha$ and HIF- $2 \alpha$ are encoded by different genes but share significant sequence homology. The relative contribution of each subunit to transcriptional regulation is not yet fully described, and they were initially thought to have largely overlapping functions. However, there is

*Correspondence: eoin.cummins@ucd.ie

School of Medicine and Medical Science, The Conway Institute, University College Dublin, Belfield, Dublin 4, Ireland emerging evidence for these oxygen-regulated transcription factors to have specific, divergent, and even opposing functions, depending on the context [7-9]. The microenvironment in which stem cells reside is complex and is subject to gradients of oxygen. Indeed, oxygen levels have been reported to direct stem cell differentiation or quiescence in different cell types [7]. Thus, stem cells and tumor stem cells can reside within a hypoxic niche and are influenced by HIFs [8]. HIF- $1 \alpha$ is described as having a dominant role in modulating WNT- $\beta$-catenin signaling, whereas HIF- $2 \alpha$ regulates OCT4, a transcription factor involved in maintaining embryonic stem cells in an undifferentiated state [9].

In a recent article in Stem Cells, Das and colleagues [10] investigate the regenerative potential of human embryonic stem cells (hESCs) with respect to HIF signaling. The central premise of this paper is that stem cells have the ability to contribute to cytoprotection of adjacent tissue through the secretion of protective growth factors and antioxidants. This 'altruistic' behavior would be attractive to exploit in the context of regenerative medicine. To achieve this in the context of tissue injury, stem cells would need to be functionally undifferentiated during hypoxia and oxidative stress. Das and colleagues demonstrate that, following exposure to hypoxia/oxidative stress (to mimic a site of injury), a small fraction of stem cells $\left(\mathrm{SSEA}^{+} / \mathrm{ABCG}^{+}\right.$) have the ability to adopt a low-p53 and high-HIF- $2 \alpha$ state for up to two weeks. This phenotype is associated with enhanced activity of Oct- 4 and Nanog and is dubbed by the authors an 'enhanced stemness' (EnSt) phenotype. The EnSt phenotype is accompanied by a high degree of selfsufficiency as measured by in vitro clonogenic assays as well as in vivo teratoma assays. In each case, the selfsufficiency of the EnSt hESCs was comparable to that of a teratoma-derived cell (Tera-2). Furthermore, conditioned media from EnSt cells had significant cytoprotective effects comparable to those of antioxidant NAC (N-acetyl cysteine).

The role of the HIFs was investigated and the authors found that HIF- $2 \alpha$-mediated suppression of p 53 is central to maintenance of the EnSt phenotype. Silencing of HIF$2 \alpha$ with siRNA (small interfering RNA) in the EnSt phenotype cells led to an almost fourfold increase in apoptosis relative to controls which was furthermore 
associated with a similar increase in p53 expression in the surviving cells. Thus, HIF- $2 \alpha$ was reported to contribute to an abnormal p53/MDM2 (a negative regulator of p53) oscillation (with EnSt cells displaying high-HIF-2 $\alpha$, low-p53, and high-MDM2 expression). HIF-1 $\alpha$ was induced by hypoxia in the model, but unlike HIF- $2 \alpha$ expression, its expression declined markedly following reoxygenation.

For regenerative medicine, the key questions arising from the work of Das and colleagues [10] are whether adult stem cells could exhibit activity similar to that of the hESCs used in the study and whether an 'enhanced stemness' phenotype could be therapeutically exploited at sites of injury in order to protect other cells from injury.

\section{Abbreviations}

EnSt, enhanced stemness; hESC, human embryonic stem cell; HIF, hypoxiainducible factor.

\section{Competing interests}

The author declares that he has no competing interests.

Published: 18 December 2012

\section{References}

1. Grocott MP, Martin DS, Levett DZ, McMorrow R, Windsor J, Montgomery HE; Caudwell Xtreme Everest Research Group: Arterial blood gases and oxygen content in climbers on Mount Everest. N Engl J Med 2009, 360:140-149.
2. Maes C, Carmeliet G, Schipani E: Hypoxia-driven pathways in bone development, regeneration and disease. Nat Rev Rheumato/ 2012, 8:358-366.

3. Eltzschig H, Carmeliet P: Hypoxia and inflammation. N Engl J Med 2011, 364:656-665.

4. Bayer C, Vaupel P: Acute versus chronic hypoxia in tumors: controversial data concerning time frames and biological consequences. Strahlenther Onkol 2012, 188:616-627.

5. Kaelin WG Jr., Ratcliffe PJ: Oxygen sensing by metazoans: the central role of the HIF hydroxylase pathway. Mol Cell 2008, 30:393-402.

6. Greer SN, Metcalf JL, Wang Y, Ohh M: The updated biology of hypoxiainducible factor. EMBO J 2012, 31:2448-2460.

7. Simon MC, Keith B: The role of oxygen availability in embryonic development and stem cell function. Nat Rev Mol Cell Biol 2008, 9:285-296.

8. Seidel S, Garvalov BK, Wirta V, von Stechow L, Schänzer A, Meletis K, Wolter M, Sommerlad D, Henze AT, Nistér M, Reifenberger G, Lundeberg J, Frisén J, Acker T: A hypoxic niche regulates glioblastoma stem cells through hypoxia inducible factor 2 alpha. Brain 2010, 133 (Pt 4):983-995.

9. Keith B, Johnson R, Simon M: HIF1a and HIF2a: sibling rivalry in hypoxic tumour growth and progression. Nat Rev Cancer 2011, 12:9-22

10. Das B, Bayat-Mokhtari R, Tsui M, Lotfi S, Tsuchida R, Felsher DW, Yeger H: HIF-2alpha suppresses p53 to enhance the stemness and regenerative potential of human embryonic stem cells. Stem Cells 2012, 30:1685-1695.

doi:10.1186/scrt143

Cite this article as: Cummins EP: HIF-2a - a mediator of stem cell altruism? Stem Cell Research \& Therapy 2012, 3:52. 